\title{
Assessing patient report of function: content validity of the Functional Performance Inventory- Short Form (FPI-SF) in patients with chronic obstructive pulmonary disease (COPD)
}

This article was published in the following Dove Press journal:

International Journal of COPD

29 August 2012

Number of times this article has been viewed

Nancy Kline Leidy'

Alan Hamilton ${ }^{2}$

Karin Becker ${ }^{3}$

'Outcomes Research, United BioSource Corporation, Bethesda, MD, USA; ${ }^{2}$ Medical Department, Boehringer Ingelheim (Canada) Ltd, Burlington, ON, Canada; ${ }^{3} \mathrm{Global}$ Health Economics and Outcomes Research, Boehringer Ingelheim $\mathrm{GmbH}$, Ingelheim am Rhein, Germany

Correspondence: Nancy Kline Leidy United BioSource Corporation, 7I0I Wisconsin Avenue, Suite 600, Bethesda, MD 208I4, USA

$\mathrm{Tel}+\mathrm{I} 30$ I 6547272

Fax +I 3016549864

Email nancy.leidy@unitedbiosource.com
Purpose: The performance of daily activities is a major challenge for people with chronic obstructive pulmonary disease (COPD). The Functional Performance Inventory (FPI) was developed based on an analytical framework of functional status and qualitative interviews with COPD patients describing these difficulties. The 65-item FPI was reduced to a 32-item short form (SF) through a systematic process of qualitative and quantitative item reduction and formatted for greater clarity and ease of use. This study examined the content validity of the reduced, reformatted form of the instrument, the FPI-SF.

Patients and methods: Qualitative cognitive interviews were conducted with COPD patients recruited from three geographically diverse pulmonary clinics in the United States. Interviews were designed to assess respondent interpretation of the instrument, evaluate clarity and ease of completion, and identify any new activities participants found important and difficult to perform that were not represented by the existing items.

Results: Twenty subjects comprised the sample; 12 (60\%) were male, 14 (70\%) were Caucasian, the mean age was $63.0 \pm 11.3$ years, $12(60 \%)$ were retired, the mean forced expiratory volume in 1 second $\left(\mathrm{FEV}_{1}\right)$ was $1.5 \pm 0.5 \mathrm{~L}$, and the mean percent predicted $\mathrm{FEV}_{1}$ was $48.4 \% \pm 13.1 \%$. Participants understood the FPI-SF as intended, including instructions, items, and response options. Two minor formatting changes were suggested to improve clarity of presentation. Participants found the content of the FPI-SF to be comprehensive, with items covering activities they felt were important and often difficult to perform.

Conclusion: These results, together with its development history and previously tested quantitative properties, suggest that the FPI-SF is content valid for use in clinical studies of COPD.

Keywords: activities of daily living, chronic pulmonary disease, COPD, functional status, health outcomes, health-related quality of life, patient-reported outcomes, physical activity

\section{Introduction}

Physical activity can be challenging for people with chronic obstructive pulmonary disease (COPD), with difficulties increasing as the severity of disease worsens over time. ${ }^{1}$ There is a growing body of evidence to suggest that both the level and intensity of daily activity are lower in people with COPD compared to healthy controls, which may be attributed to poorer exercise tolerance, difficulties with usual day-today activities, and fear or concern related to pushing themselves to higher levels. ${ }^{2-8}$ 
Given the evidence linking activity to general health, ${ }^{9}$ hospital admissions for exacerbation, ${ }^{10-12}$ and mortality, ${ }^{11,13}$ important goals of treatment and key outcomes in clinical trials of patients with COPD include improving exercise capacity, easing the performance of daily activities, and increasing levels of physical activity. ${ }^{14-17}$

The Functional Performance Inventory (FPI) is a patientreported outcome (PRO) measure developed to quantify the ease/difficulty with which COPD patients perform daily activities. ${ }^{18,19}$ Although predating the United States Food and Drug Administration (FDA) Guidance on PRO measures, ${ }^{20}$ methods used to develop the 65-item FPI were consistent with this guidance and good research practices in instrument development. ${ }^{21-23}$ These practices include the use of an underlying theoretical framework, ${ }^{24-26}$ qualitative elicitation and evaluative cognitive interviews in the target population to inform instrument content and structure, ${ }^{27,28}$ and input from experts to assure clinical relevance. ${ }^{18}$ This maximizes content validity, defined as the extent to which an instrument measures important aspects of the target concept. ${ }^{21,23}$ The quantitative phase of instrument development includes item-level analyses, an assessment of measurement structure through factor analyses, estimation of score internal consistency and test-retest reliability, and tests of score validity, including sensitivity to change. Reliability, validity, and responsiveness of FPI scores in subjects with COPD have been demonstrated in cross-sectional and longitudinal studies. ${ }^{18,19,29,30}$

To reduce respondent burden, a 32-item short form (FPI-SF) was developed using a systematic process of qualitative and quantitative item reduction. ${ }^{31}$ To optimize content validity and score equivalence with the longer form, qualitative patient data, clinician ratings, and quantitative validation data from the FPI development work were used to identify items for elimination and to test the properties of the short form. ${ }^{31}$ Although the FPI-SF has been used in several studies, providing evidence of score reliability, validity, and responsiveness, ${ }^{32-34}$ content validity has not been examined independently of the original development work. The need for additional qualitative research to assure content validity of a revised instrument is consistent with good research practices. ${ }^{23}$ Specifically, cognitive interviewing methodology is used to identify any areas of respondent misunderstanding or confusion and uncover missing content that is highly relevant and important to patients and therefore essential to include in the instrument. Results are used to adjust the instrument or, if no changes are needed, assure users that this important type of validity has been addressed. $22,23,35$

The purpose of this study was to evaluate the content validity of the FPI-SF and refresh the original qualitative work through cognitive interviews with a sample of patients with COPD experiencing the disease under current treatment and social conditions. Results were used to make any changes needed to improve the instrument or to assure previous and new users of its content validity and suitability for clinical research. The information also provides updated insight into the types of activities that are problematic for patients with COPD to guide further work in this area.

\section{Material and methods}

This cross-sectional, qualitative study involved one-onone, in-person cognitive interviews with men and women with COPD. Subject enrollment continued to the point of "saturation," ie, until no new information or concepts emerged with each successive interview. Sample size for qualitative studies of this nature can be as small as 7 to 10 to confirm that patients understand items as intended, while larger samples are necessary for more complex measures. ${ }^{22,35}$ In this case, a target sample size of 20 was selected to assure saturation.

\section{Sample}

Patients were identified, screened, and recruited from three geographically diverse pulmonary clinics in the United States. Clinic staff identified potential subjects using patient records and telephone contact or during scheduled office visits. All recruitment procedures complied with current Health Insurance Portability and Accountability Act of 1996 regulations, the recruitment locations (ie, the three clinical sites) were approved by a central institutional review board (IRB), and all study procedures adhered to the IRB-approved protocol and tenets of the Declaration of Helsinki.

Patients were eligible for the study if they were male or female, 40 years of age or older, were a current or former cigarette smoker with a smoking history of at least 10 pack-years, had a current medical diagnosis of moderate (Global Initiative for Chronic Obstructive Lung Disease [GOLD] Stage II), severe (GOLD Stage III), or very severe (GOLD Stage IV) COPD (including chronic bronchitis and/ or emphysema), had a post-bronchodilator forced expiratory volume in 1 second $\left(\mathrm{FEV}_{1}\right)<80 \%$ of the predicted value 
and $\mathrm{FEV}_{1} /$ forced vitality capacity $(\mathrm{FVC})<0.70$ documented in the previous year, were able to participate in a one-on-one in-person interview, were able to read, speak, and understand English and complete all study assessments, and were willing and able to provide written informed consent prior to the interview.

Patients were excluded from the study if they met any of the following criteria: a clinically relevant medical or psychiatric condition which, in the opinion of the site investigator and/or site study coordinator, would interfere with completing the study, including, but not limited to, sensory problems, cognitive impairment, acute mental illness or inadequately treated depression or anxiety, COPD exacerbation or unexpected visit to the clinic, emergency room (ER), or hospital and/or prescription medication to treat an exacerbation in the previous four weeks, diagnosis of asthma, respiratory disease other than COPD that contributed to ventilatory limitation, or had a condition other than COPD that, in the opinion of the investigator, substantially interfered with or reduced the patient's functional performance, ie, the ease with which they performed daily physical activities.

The study protocol was approved by the Ethical and Independent Review Services, a central IRB, and each patient provided written informed consent prior to the start of the interview.

\section{Measures}

\section{FPI-SF}

The FPI-SF is a 32-item self-administered questionnaire to assess the level of difficulty respondents have with physical activities across six domains or subscales, including body care (five items), maintaining the household (eight items), physical exercise (five items), recreation (five items), spiritual activities (four items), and social interaction (five items). For ease of use, activities assessed are organized according to these domains. For each item/activity, respondents are asked to rate how difficult the activity is for them to perform on a simple three-point scale: "no difficulty," "some difficulty," or "much difficulty." If respondents do not perform an activity, they can select one of two options: "don't do for health reasons" or "choose not to." The format of the items, as presented in the questionnaire, is shown in Figure 1.

Total and subscale scores range from 0 to 3 , with higher scores indicating higher levels of functional activity within and across activity domains. Subjects receive no (0) points for activities they do not perform, whether for health or other reasons; performing an activity with much difficulty receives a score of 1 , with some difficulty receives a 2 , and with no difficulty receives a 3 . Subscale scores are expressed as mean values, with an $80 \%$ completion rate required for calculation. The total score is the mean across the six domains. Respondents engaged in many activities with no difficulty will score high on the FPI, while those who perform few activities with much difficulty will score low on the instrument.

\section{Sociodemographic and clinical characteristics}

To characterize the sample, participants completed a brief questionnaire booklet at the close of the interview with a sociodemographic questionnaire and the St George's Respiratory Disease Questionnaire (SGRQ). The SGRQ is a 50-item respiratory-specific health status measure assessing symptoms (frequency and severity), activity (activities that cause or are limited by breathlessness), and impacts (social functioning, psychological disturbances resulting from airways disease) of COPD. Higher scores on the SGRQ indicate poorer health status.

The clinical site staff completed a form providing information on pulmonary function, GOLD stage, and clinicianrated dyspnea using the modified Medical Research Council (mMRC) dyspnea scale.

\section{Procedures}

\section{Cognitive interviews}

A semistructured cognitive interview guide was used to elicit information regarding the comprehensiveness, clarity, and interpretation of the questionnaire's instructions, items and response options, ease of completion, relevancy of the items, formatting (eg, design and placement of instructions, font, placement of items on page), and identification of new concepts (eg, functional areas or activities that patients consider relevant, but which are not represented by existing items). The guide was structured such that the participant would read and respond to one section of the FPI-SF at a time, with each completion followed by interview questions related to that section of the questionnaire. The interview guide was not used as a verbatim script; probes and questions were changed during the course of the interviews based on individual patient responses to assure accurate and comprehensive data.

All interviews were conducted by a research professional experienced in qualitative interviewing techniques and trained on the study's background, objectives, methods, and procedures. Each interview lasted 60 to 90 minutes, took place in a private and confidential space at the clinical 


\begin{tabular}{lccc|cc} 
& \multicolumn{3}{c|}{ DO with ... } & \multicolumn{2}{c}{ DON'T DO because ... } \\
\cline { 2 - 7 } Body care & $\begin{array}{c}\text { No } \\
\text { difficulty }\end{array}$ & $\begin{array}{c}\text { Some } \\
\text { difficulty }\end{array}$ & $\begin{array}{c}\text { Much } \\
\text { difficulty }\end{array}$ & $\begin{array}{c}\text { Health } \\
\text { reasons }\end{array}$ & $\begin{array}{c}\text { Choose } \\
\text { not to }\end{array}$ \\
\hline Dressing and undressing & 1 & 2 & 3 & $\square$ & $\square$ \\
\hline Showering or bathing & 1 & 2 & 3 & $\square$ & $\square$ \\
\hline Caring for your feet & 1 & 2 & 3 & $\square$ & $\square$ \\
\hline Washing your hair & 1 & 2 & 3 & $\square$ & $\square$
\end{tabular}

Figure I Presentation format for the FPI-SF. ${ }^{a}$

Notes: allustration presents one of six domains comprising the FPI-SF.

Abbreviation: FPI-SF, Functional Performance Inventory-Short Form.

sites, was audio-recorded (with patient permission), and was transcribed and de-identified for analyses.

\section{Analyses}

Analyses were performed using ATLAS.ti (v. 5.0; Scientific Software Development, Berlin, Germany), a software package designed to facilitate the organization, coding, and analyses of qualitative data.

Data were coded by trained coders using a content analysis approach, organized and analyzed based on how respondents interpreted each component of the measure (instructions, items, response options, recall period), how and why they selected their response to each item, and any difficulties or areas of confusion respondents had with the instrument (terminology or vocabulary, format). Coding also included how representative, relevant, and/or important each activity was to the respondents and any indications that important activities were omitted. Saturation, defined as the point at which no substantially new or novel information emerges, ${ }^{22}$ was documented.

Sample demographic and clinical characteristics were summarized using descriptive statistics.

\section{Results}

\section{Patient sample}

Twenty COPD patients participated in one-on-one interviews from February 17, 2011 through March 16, 2011. Participant demographic characteristics are shown in Table 1, with clinical characteristics summarized in Table 2. FPI-SF total and profile scores are shown in Figure 2. Respondents reported the highest functional performance in body care activities and the lowest in physical exercise and spiritual activities.
Saturation was reached early in the study, with each interview providing supportive and insightful information regarding the daily activities of people with varying sociodemographic and clinical characteristics.

\section{Instructions and response options}

All participants $(\mathrm{N}=20 ; 100 \%)$ understood the FPI-SF instructions and response options and were able to accurately describe them in their own words.

\section{Items/activities}

Sample quotations from the data are shown in Table 3. These quotations represent patient descriptions of the meaning of a given domain or item in the FPI-SF or the ease or difficulty

Table I Sample demographic characteristics

\begin{tabular}{ll}
\hline Characteristic & Sample (N = 20) \\
\hline Age (years), mean (SD) [range] & $63.0(11.3)[49-86]$ \\
Gender: male, N (\%) & $12(60.0 \%)$ \\
Racial background, N (\%) & $14(70.0 \%)$ \\
$\quad$ White & $6(30.0 \%)$ \\
Black/African American, Hispanic, & \\
Asian/Hispanic & \\
Living situation, N (\%) & $15(75.0 \%)$ \\
With partner/spouse/family & $5(25.0 \%)$ \\
Alone & \\
Employment status, N (\%) & $5(25.0 \%)$ \\
$\quad$ Employed, full- or part-time & $2(10.0 \%)$ \\
Unemployed & $13(65.0 \%)$ \\
Retired or disabled & \\
Education, N (\%) & $6(30.0 \%)$ \\
High school or equivalent or less & $7(35.0 \%)$ \\
Associate degree, technical, or trade school & $7(35.0 \%)$ \\
College & \\
\hline
\end{tabular}

Abbreviation: SD, standard deviation. 
Table 2 Sample clinical characteristics

\begin{tabular}{|c|c|}
\hline Characteristic & Sample $(\mathbf{N}=\mathbf{2 0})$ \\
\hline Years since diagnosis, mean (SD) [range] & $9.8(7.0)[1-26]$ \\
\hline \multicolumn{2}{|l|}{ Pulmonary function } \\
\hline $\mathrm{FEV}_{1}[\mathrm{~L}]$, mean (SD) [range] & $1.4(0.5)[0.5-2.1]$ \\
\hline FEV,$\%$ predicted, mean (SD) [range] & $48.4(13.1)[27.0-72.0]$ \\
\hline $\mathrm{FEV}_{1} / \mathrm{FVC}$, mean (SD) [range] & $30.2(28.7)[0.3-68.9]$ \\
\hline \multicolumn{2}{|l|}{ GOLD COPD severity level, $\mathrm{N}(\%)$} \\
\hline GOLD II - moderate & $9(45.0 \%)$ \\
\hline GOLD III - severe & $9(45.0 \%)$ \\
\hline GOLD IV - very severe & $2(10.0 \%)$ \\
\hline Smoking history, $\mathrm{N}(\%)$ current smoker & $9(45.0 \%)$ \\
\hline Pack-years, mean (SD) & $48.9(28.5)$ \\
\hline \multicolumn{2}{|l|}{ Clinician-rated dyspnea (mMRC), N (\%) } \\
\hline No breathlessness except with strenuous exercise & $2(10.0 \%)$ \\
\hline Breathlessness hurrying on the level or slightly uphill & $8(40.0 \%)$ \\
\hline Walks slower than people due to breathlessness or stops when walking at own pace on the level & $7(35.0 \%)$ \\
\hline Stops for breath after walking 100 yards or a few minutes on the level & $2(10.0 \%)$ \\
\hline Too breathless to leave the house or breathless dressing or undressing & $\mathrm{I}(5.0 \%)$ \\
\hline \multicolumn{2}{|l|}{$\mathrm{SGRQ}^{\mathrm{a}}$} \\
\hline Total score & $42.05(18.13)$ \\
\hline Symptom scale & $54.26(25.66)$ \\
\hline Activity scale & $54.70(24.15)$ \\
\hline Impact scale & $28.94(17.98)$ \\
\hline
\end{tabular}

Notes: aRange: 0 to 100; higher scores indicate poorer health status.

Abbreviations: COPD, chronic obstructive pulmonary disease; FEV , forced expiratory volume in I second; FVC, forced vitality capacity; GOLD, Global Initiative for Chronic Obstructive Lung Disease; mMRC, Modified Medical Research Council Dyspnea Scale; SD, standard deviation; SGRQ, St George's Respiratory Questionnaire.

with which they perform the activity. In the presentation of results below, all participants $(\mathrm{N}=20 ; 100 \%)$ understood an item as intended unless otherwise specified. The descriptions provide insight into how participants interpreted the items and the nature of the ease or difficulty performing the activity in their daily lives.

\section{Body care}

Participants provided appropriate examples of body care, such as showering or dressing (40\%), or described "body care" as "taking care of oneself" (35\%) or "hygiene" (30\%). No participant selected "don't do" body care activities for health reasons; all participants selected "some" or "much" difficulty for at least one of these activities.

\section{Dressing and undressing}

Most participants reported no difficulties dressing and undressing. Of the three participants who had trouble with this task, two reported experiencing breathlessness, and one reported lack of energy.

\section{Showering or bathing}

Of the five participants reporting difficulty with this activity, one described experiencing breathlessness and a back problem that made it difficult to stand in the shower for a long period of time, and one described difficulties getting in and out of the bathtub.

\section{Caring for your feet}

Four participants reporting difficulty described breathlessness or breathing problems with this activity. Three other participants stated that they had other health conditions (ie, being overweight, nerve damage, athlete's foot) that made it difficult to care for their feet.

\section{Washing your hair}

The two patients selecting "chose not to" perform this activity attributed this choice to not having hair.

\section{Shaving or applying makeup}

Two participants had difficulty with this activity, both due to breathlessness.

\section{Maintaining the household}

Participants described this activity category as housework/ household chores $(30 \%)$ or provided appropriate examples such as "cleaning" (65\%), "cooking" or "grocery shopping" $(20 \%)$, yard work or mowing the lawn (15\%), taking out trash $(10 \%)$, getting the mail (5\%), and laundry (5\%). All participants except one reported that they do all of the tasks 


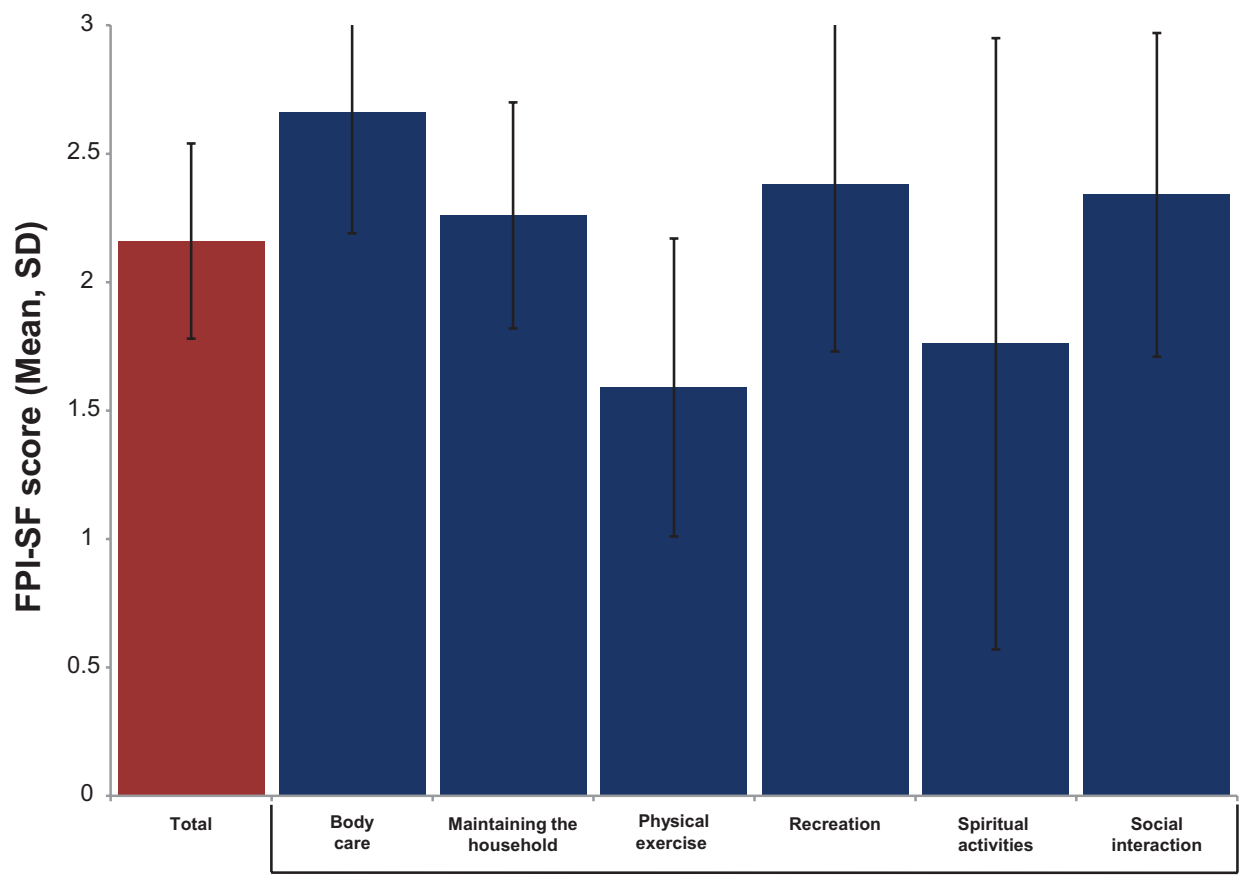

\section{Domain}

Figure 2 FPI-SF total and profile scores. ${ }^{a}$

Notes: ${ }^{a} \mathrm{~N}=20$; higher scores indicate higher levels of performance.

Abbreviations: FPI-SF, Functional Performance Inventory - Short Form; SD, standard deviation.

listed under "maintaining the household," although some with "some difficulty" or "much difficulty." The remaining participant reported not "mowing the lawn, shoveling snow, raking, or heavy gardening" due to health reasons.

\section{Groceries and meals}

\section{Preparing meals/cooking}

Two participants reported difficulty and described breathlessness or difficulty walking around the kitchen when trying to do this activity.

\section{Grocery shopping}

Participants reporting problems with this activity described breathlessness and difficulties carrying groceries, walking in the grocery store, or keeping up with their spouse.

\section{Carrying groceries}

More than half of participants described breathlessness when carrying groceries. Several participants reported being unable to carry the groceries or having trouble with stairs.

\section{Activities around the house or apartment}

All were able to provide specific, appropriate examples, including cleaning or maintaining their homes (90\%), yard work (15\%), or taking care of pets $(15 \%)$. Most participants reported "some difficulty" or "much difficulty" with each of these activities.

\section{Going to appointments (such as doctors or dentists)}

When asked to describe the meaning of this task, participants described the process of traveling to the appointment $(65 \%)$ and/or getting ready for the appointment $(30 \%)$.

\section{Physical exercise}

Participants described this domain of activity as "doing my exercise," "running, lifting weights," and "staying in shape."

\section{Regular stretching, moving, or lifting light weights}

Just over half of the participants responded that they did not have any difficulties with regular stretching, moving, or lifting light weights $(\mathrm{N}=11 ; 55 \%)$, while the remaining participants $(\mathrm{N}=9 ; 45 \%)$ reported having difficulties. Of these, five reported difficulty due to breathlessness, two reported only doing a little bit or doing it slowly, two reported getting tired or their muscles getting tired, and one was unable to lift objects. One participant reported that the exercise routine was challenging. 
Table 3 Representative quotations by item (activity) and code ${ }^{a}$

\author{
Domain \\ Activity/item ( $\mathrm{N}$ reporting some or much difficulty) \\ Body care \\ Dressing and undressing (4) \\ Showering or bathing (5) \\ Caring for your feet (2)
}

\section{Maintaining the household \\ Groceries and meals \\ Preparing meals/cooking (2) \\ Grocery shopping (6)}

Carrying groceries (15)

Activities around the house or apartment

Vacuuming or sweeping (I3)

Moving furniture, changing sheets, or washing windows (16)

Going to appointments (such as doctors or dentists) (I3)

\section{Physical exercise}

Walking up and down a flight of stairs (16)

Short walks around the neighborhood or mall (9)

\section{Recreation: activities for personal pleasure \\ Taking vacations (I)}

Activities away from the house or apartment Indoor activities such as shopping or museums (6)

Going to the movies (0)

Activities around the house or apartment

Sitting outside (0)

Reading ( $)$

\section{Spiritual activities}

Going to religious ceremonies (I)

Visits from spiritual friends or teachers $(0)$
Code: Representative respondent quotation (Selected items - full list available on request)

Difficulty: I have a hard time putting my clothes on ... because l'm out of breath and I have to do one leg at a time. (ID I03)

Difficulty: It's like running a marathon. I mean, it's hard work to take a shower. (ID 105)

Difficulty: The reason for that being, um, breathing can be an issue bending down .... Um, if I bend down too quickly, I can-I find myself getting out of breath very quickly and it's uncomfortable. (ID IOI)

Difficulty: The heat makes me get short of breath every once in a while. I just have to back off and slow down. I love to cook. (ID 205)

Difficulty: You're constantly in motion around the grocery store ... I get anxious if I don't have my albuterol with me because I can get out of breath quickly in a grocery store, um, so, yes, uh, some difficulty. (ID IOI)

Difficulty: I do have some difficulty with my breathing if I have a lot of heavy groceries to carry in. (ID 203)

Difficulty: Because I get short of breath doing it, pushing the vacuum cleaner or sweeping, using a broom, even mopping. (ID 205)

Meaning: Changing the furniture around in the room or, moving furniture to sweep or vacuum or mop underneath them or behind them, changing sheets is just that, stripping my bed, folding bedding and, putting clean bedding on it. And, washing windows, that means Windex and paper towels for me. (ID 203)

Meaning: Just getting in my car and-and making my appointments, being at the appropriate doctor at the appropriate time ... driving there, parking, going into the office, and, going back to see the doctor or dentist or what, you know. (ID 203)

Difficulty: Going up stairs, that-and every time I have to go upstairs at work for the two flights, I really, really have to think about it and then pace myself ... and I just take my time, and if I have to stop halfway up, I'll stop, you know, and that's-so, yes, going upstairs can be a challenge. (ID IOI)

Difficulty: Well, because some times, like l'm walking I get out of breath, you know. When I walk anywhere, I'm out of breath all the time, regardless ... I try as much as I can to walk around the malls, around a grocery store or whatever ... but I'm still out of breath. (ID 103)

Difficulty: Yeah, I don't have no difficulty doing it. I can get in the car and drive. (ID 205)

Difficulty: There is lots of things I can't do. I can get there but when it comes to walking around and standing and going up and down stairs and stuff that's hard on me so I usually go with them and I find a place to sit down. (ID 20I)

Difficulty: I don't feel any difficulties going to a movie. It's just, you know, get in my car, ride to the theater and go in and sit down and watch the movie. It doesn't seem to cause me any difficulty. (ID 203)

Difficulty: No problems whatsoever. Um, we just-we sit on the back porch quite often. (ID IOI)

Difficulty: Yeah. Activities like that, reading or journaling and all, they do not cause me any difficulty with my breathing. (ID 203)

Meaning: Christenings, uh, weddings, baptisms, that type of thing. (ID IOI)

Meaning and difficulty: Visit from spiritual-spiritual friends and teachers - and teachers, uh, 'not difficult' because, uh, they come and they sit around my house and we pray and we talk spiritually things. (ID I02) 
Table 3 (Continued)

Social interaction: family and friends

Dinner, cards, bingo or other activity: in your home (I)

Helping in the care of children (8)

Distant or overnight travel to visit others (2)
Meaning: Having your family over or going over to see them and, uh, having your friends over. And, you know, just having like a picnic or cookout or something like. (ID 303)

Difficulty: Sometimes I get out of breath, you know, just having to get up and get drinks or - or move around the kitchen, doing more than I usually do. (ID 205)

Difficulty: Keeping up with children can cause me some difficulty at times, um, especially if I have to get down on the floor and up off the floor a lot or, uh, if it's a small child that I have to carry around a good bit. That can cause me a little difficulty. (ID 203)

Difficulty: Because I think that it would involve carrying luggage ... that may affect my breathing somewhat, in reference to my COPD. (ID 107)

Note: ${ }^{\text {F}}$ For space reasons, not all items are shown.

\section{Walking up and down a flight of stairs}

The most frequently reported reason for difficulty with this activity was breathlessness $(\mathrm{N}=10 ; 50 \%)$. One patient reported wheezing, another reported having to go slowly, and another reported difficulty when carrying something.

\section{Short walks around the neighborhood or mall}

Breathlessness was the most frequently cited reason for difficulty with this activity (30\%), followed by getting tired, wheezing, and coughing, mentioned by one patient each.

\section{Long fast walks (more than 20 minutes)}

The most frequently cited reason for difficulty with this activity was breathlessness (35\%), followed by getting tired and needing to rest, and coughing, which were mentioned by one patient each.

\section{Activities such as swimming or bicycling}

All participants understood swimming and bicycling to be examples of physical activity. When asked to provide other activities similar to swimming or bicycling, respondents offered "basketball," "playing soccer," "rowing on a row machine or in a canoe," "other kinds of physical exercise, like jogging," "bowling," and a "horseback ride." The most frequently cited reason for difficulty with activities was breathlessness $(\mathrm{N}=5 ; 25 \%)$.

\section{Recreation: activities for personal pleasure}

Participants described this activity label as fun activities or special events, providing examples that included vacations $(25 \%)$, reading $(15 \%)$, movies $(10 \%)$, shopping $(5 \%)$, and relaxing at home $(5 \%)$.

\section{Taking vacations}

The majority of participants $(70 \%)$ reported having no difficulty taking vacations.

\section{Activities away from the house or apartment}

All participants understood this item as intended and were able to provide descriptions of activities away from the home.

Indoor activities such as shopping or museums

Participants provided examples of shopping, including going to the grocery store, hardware store, or another type of store or mall $(65 \%)$ and visiting museums, tourist attractions, or the zoo (55\%). Six (30\%) reported that they had difficulty, including three that reported getting out of breath and one each reported needing to go slow, getting tired easily, or trouble walking.

\section{Going to the movies}

Most participants reported no difficulty or chose not to perform this activity.

\section{Activities around the house or apartment}

Most participants understood the phrase as intended (85\%), describing it as sitting outside (45\%), reading (40\%), watching TV or listening to the radio (30\%), hobbies $(15 \%)$, and gardening $(5 \%)$.

\section{Sitting outside}

Most participants reported that they did not have any difficulties with this activity.

\section{Reading}

Most participants reported that they did not have any difficulties with "reading." One participant reported difficulty due to macular degeneration.

\section{Spiritual activities}

Descriptions of spiritual activities included going to church or religious services $(60 \%)$, religious reading, meditation or prayer (20\%), and religious ceremonies (5\%). 


\section{Attending religious services}

All participants described "attending religious services" as going to "church" or "synagogue." Half of the participants reported that they selected the "choose not to" response because they do not attend religious services. One subject reported difficulty with attending religious services due to trouble breathing at church.

\section{Going to religious ceremonies}

Examples of this activity provided by participants included "christenings," "weddings," "baptisms," or "funerals."

\section{Personal reading, meditation or prayer}

Participants described this activity as talking to God (45\%) and/or reading the bible or other religious readings $(60 \%)$.

\section{Visits from spiritual friends or teachers}

Most participants (85\%) understood the phrase as intended.

\section{Social interaction: family and friends}

Dinner, cards, bingo or other activity: in your home

Examples of this activity category offered by participants included having guests over for meals, watching television, or visiting with friends at the subject's home.

\section{Going to the store, giving rides, doing repairs or other favors}

Additional examples of this activity included cooking or taking care of children. Of those indicating they had difficulty with this activity, three described experiencing breathlessness, two having trouble raising their arms over their head, and two needing to go slowly.

\section{Helping in the care of children}

Difficulties with this activity included not being able to run after children (20\%) and one patient reported having difficulty carrying children and bending down to pick them up.

\section{Distant or overnight travel to visit others}

Two patients reported having difficulty with this activity; one cited breathing problems when carrying luggage and the other needing to stop periodically and stretch.

\section{Formatting and additional comments}

Participants were asked about the format of the FPI-SF questionnaire, including placement of instructions and questions, clarity of the print, and the placement of answer choices on the questionnaire. Sixteen participants indicated they liked the format and had no suggestions for added clarity or ease of completion. Four participants suggested two formatting changes that were made to improve clarity: (1) move "getting around town" to the line before "going to appointments (such as doctors or dentists)," and (2) editorial changes to the instructions for checking the fourth and fifth boxes in the scale (don't do for "health reasons" or "choose not to").

\section{Content coverage}

All participants found the activities included in the FPI-SF to be comprehensive, representing activities they felt were important to them and either were or could be difficult to perform. No concepts were raised that had not appeared in the qualitative research forming the basis of the FPI. ${ }^{27}$ In situations in which additional candidate activities were proposed, participants were able to classify the activities in the context of other similar activities. For example, one participant indicated going to school was another indoor activity away from home and classified this as similar to going to the movies. Another offered going to the zoo and to a casino as additional activities away from the house or apartments, similar to going shopping or to the movies. Working on the computer was equated with reading.

Several participants suggested adding a question related to sexual activity, a concept identified during the original elicitation interviews. When subsequent respondents were queried about including an item addressing this (at the end of the interview if the activity was not mentioned), responses were mixed, with several respondents indicating this was not needed or that they would not respond. During development of the FPI, an item addressing sexual activity was included in the item pool, but was dropped during item reduction due to substantial missing data.

\section{Discussion}

The low levels of physical activity generally observed in people with COPD may be due in part to the difficulties they experience as they attempt to perform daily activities that they need and want to perform. ${ }^{36}$ These difficulties can have a negative reinforcement effect, leading to a downward spiral of inactivity and poorer quality of life, morbidity, and mortality outcomes. ${ }^{1,4,10-13,37}$ Many interventions, including pharmacologic, rehabilitation, behavioral, and/or exercise programs, are designed to help patients boost their exercise capacity, reduce difficulties with daily functioning, and increase their levels of physical activity to minimize or reverse the longer term adverse effects of a sedentary lifestyle. The FPI-SF 
was designed to standardize and quantify patient-reported ease or difficulty with which patients perform a range of daily activities important to them. In descriptive studies and clinical trials, these data can complement and extend results on exercise capacity and the amount and intensity of daily activity to provide a comprehensive understanding of physical activity in patients with COPD.

This study was conducted to ascertain whether content validity of the FPI was adversely affected during the development of the FPI-SF or over time, ie, whether activity types, difficulties, or importance, had qualitatively changed since the first development work. If adjustments in the instrument were required, this would be done immediately before the instrument was used in further research. If content validity was supported, this information would assure users that the measure is suitable for clinical research in COPD.

The men and women who participated in this study represented a range of patients with this condition. They were ethnically and sociodemographically diverse, including participants who were working, retired, and high school and college graduates. Participants had moderate to severe airflow obstruction and varied levels of dyspnea. SGRQ scores indicated moderate levels of health status impairment, on average, with a mean and range consistent with previous studies of patients with moderate to severe COPD. The reported mean values on the FPI-SF for this sample were descriptively higher than those in the original validation survey, ${ }^{31}$ indicating that patients were performing more activities with less difficulty. The profile patterns were identical across the two samples, with highest functioning in body care activities and lowest functioning in physical exercise.

Consistent with the initial qualitative work underlying the development of the FPI, most participants in the present study reported difficulty with high exertion activities, such as moving furniture, changing sheets or washing windows, walking up and down a flight of stairs, vacuuming or sweeping, and carrying groceries. Across activities, shortness of breath was a clear theme, with participants explaining their difficulty ratings with specific examples of their breathlessness experience. Previous research describing the effects of COPD on daily activities have shown similar results. ${ }^{38}$

Results supported the content validity of the FPI-SF for quantifying self report of functional performance in patients with COPD. Participants understood the instructions, items, and response options as intended and no new content was uncovered, indicating that the instrument is content valid in this target population. Two minor formatting changes were made to the questionnaire for added clarity of presentation.

Several studies have used the FPI-SF, providing insight into areas of functional difficulty for patients with COPD, factors that contribute to these difficulties, and candidate interventions. For example, Wall (2007) found that depression, disease severity, gender, and age accounted for nearly half $(46 \%)$ of the variance in functional performance (FPI-SF score) observed in a sample of 119 patients with moderate to severe COPD. ${ }^{34}$ Results of a randomized pilot study reported by Donesky-Cuenco and colleagues ${ }^{32}(\mathrm{~N}=29)$ suggested that a 12-week yoga program for patients with moderate to severe COPD may help patients improve their walking capacity, reduce dyspnea-related distress, and improve functional performance, with the FPI-SF showing a statistically significant between-group treatment effect $(P=0.04)$ with a large effect size (0.79). Given the strong correlation between FPI and FPI-SF score shown previously (0.98), ${ }^{31}$ insight can also be gleaned from studies using the long form of the instrument. A 3-year longitudinal study by Kapella et $\mathrm{al}^{30}$ of patients with COPD $(\mathrm{N}=108)$, for example, showed significant declines in functional performance over time associated with declines in spirometry, exercise capacity, and muscle strength.

For men and women with COPD, important goals of treatment are to improve functional performance, reverse sedentary lifestyles, and increase or maintain activity level. ${ }^{14}$ As a measure of functional performance, the FPI-SF can be used to identify the daily activities patients find most difficult or the individual patients with the greatest difficulty, to inform targeted treatment. It can also be used to identify high performers for research related to the characteristics of these individuals, providing insight into interventions to help them maintain this performance over time or uncover new interventions to help those finding daily activity difficult. The FPI-SF was also designed to serve as an outcome measure in clinical trials evaluating the effect of interventions on this important aspect of physical activity.

\section{Conclusions}

Results of this study support the content validity of the FPI-SF and indicate that this PRO instrument is suitable for use in clinical studies examining functional performance in patients with COPD.

\section{Acknowledgments}

This study was funded by Boehringer Ingelheim. The authors would like to thank Julie McCormack for assisting with 
protocol development and data collection, Erin Sullivan for conducting the interviews and assisting with data analyses, Louisa Wahala for assisting with data analyses, and Kathryn Miller for assistance with manuscript formatting for submission. The FPI-SF ( ${ }^{\circledR} 1999$, Nancy Kline Leidy) and user manual may be obtained from Dr Leidy.

\section{Disclosure}

Nancy Kline Leidy is employed by the United BioSource Corporation (UBC), which provides consulting and other research services to pharmaceutical, device, government and non-government organizations. In this salaried position, Dr Leidy works with a variety of companies and organizations. She receives no payment or honoraria directly from these organizations for services rendered. Alan Hamilton and Karin Becker are fulltime employees of Boehringer Ingelheim. The authors have no other conflicts to disclose.

\section{References}

1. Watz H, Waschki B, Meyer T, Magnussen H. Physical activity in patients with COPD. Eur Respir J. 2009;33(2):262-272.

2. Bossenbroek L, de Greef MH, Wempe JB, Krijnen WP, Ten Hacken NH. Daily physical activity in patients with chronic obstructive pulmonary disease: a systematic review. COPD. 2011;8(4):306-319.

3. Eliason G, Zakrisson AB, Piehl-Aulin K, Hurtig-Wennlof A. Physical activity patterns in patients in different stages of chronic obstructive pulmonary disease. COPD. 2011;8(5):369-374.

4. Hill K, Goldstein RS. Limited functional performance in chronic obstructive pulmonary disease: nature, causes and measurement. $C O P D$. 2007;4(3):257-261.

5. Pitta F, Troosters T, Spruit MA, Probst VS, Decramer M, Gosselink R. Characteristics of physical activities in daily life in chronic obstructive pulmonary disease. Am J Respir Crit Care Med. 2005;171(9): 972-977.

6. Sandland CJ, Singh SJ, Curcio A, Jones PM, Morgan MD. A profile of daily activity in chronic obstructive pulmonary disease. J Cardiopulm Rehabil. 2005;25(3):181-183.

7. Troosters T, Sciurba F, Battaglia S, et al. Physical inactivity in patients with COPD, a controlled multi-center pilot-study. Respir Med. 2010;104(7):1005-1011.

8. Vorrink SN, Kort HS, Troosters T, Lammers JW. Level of daily physical activity in individuals with COPD compared with healthy controls. Respir Res. 2011;12:33.

9. Garcia-Aymerich J, Serra I, Gomez FP, et al. Physical activity and clinical and functional status in COPD. Chest. 2009;136(1):62-70.

10. Garcia-Aymerich J, Farrero E, Felez MA, Izquierdo J, Marrades RM, Anto JM. Risk factors of readmission to hospital for a COPD exacerbation: a prospective study. Thorax. 2003;58(2):100-105.

11. Garcia-Aymerich J, Lange P, Benet M, Schnohr P, Anto JM. Regular physical activity reduces hospital admission and mortality in chronic obstructive pulmonary disease: a population based cohort study. Thorax. 2006;61(9):772-778.

12. Pitta F, Troosters T, Probst VS, Spruit MA, Decramer M, Gosselink R. Physical activity and hospitalization for exacerbation of COPD. Chest. 2006;129(3):536-544.

13. Waschki B, Kirsten A, Holz O, et al. Physical activity is the strongest predictor of all-cause mortality in patients with COPD: a prospective cohort study. Chest. 2011;140(2):331-342.

14. Bourbeau J. Activities of life: the COPD patient. COPD. Jun 2009;6(3): 192-200.
15. Cooper CB. Exercise in chronic pulmonary disease: aerobic exercise prescription. Med Sci Sports Exerc. 2001;33(7 Suppl):S671-S679.

16. Cindy Ng LW, Mackney J, Jenkins S, Hill K. Does exercise training change physical activity in people with COPD? A systematic review and meta-analysis. Chron Respir Dis. 2012;9(1):17-26.

17. Troosters T, Gosselink R, Janssens W, Decramer M. Exercise training and pulmonary rehabilitation: new insights and remaining challenges. Eur Respir Rev. 2010;19(115):24-29.

18. Leidy NK. Psychometric properties of the functional performance inventory in patients with chronic obstructive pulmonary disease. Nurs Res. 1999;48(1):20-28.

19. Leidy NK, Knebel A. Clinical validation of the Functional Performance Inventory in patients with COPD. Respir Care. 1999;44(8): 932-939.

20. Food and Drug Administration. Guidance for industry on patient-reported outcome measures: use in medical product development to support labeling claims. Federal Register. 2009;74(235): 65132-65133.

21. Patrick DL, Burke LB, Gwaltney CJ, et al. Content validity establishing and reporting the evidence in newly developed patientreported outcomes (PRO) instruments for medical product evaluation: ISPOR PRO good research practices task force report: part 1-eliciting concepts for a new PRO instrument. Value Health. 2011;14(8): 967-977.

22. Patrick DL, Burke LB, Gwaltney CJ, et al. Content validity - establishing and reporting the evidence in newly developed patient-reported outcomes (PRO) instruments for medical product evaluation: ISPOR PRO Good Research Practices Task Force report: part 2 - assessing respondent understanding. Value Health. 2011;14(8):978-988.

23. Rothman M, Burke L, Erickson P, Leidy NK, Patrick DL, Petrie CD. Use of existing patient-reported outcome (PRO) instruments and their modification: the ISPOR Good Research Practices for Evaluating and Documenting Content Validity for the Use of Existing Instruments and Their Modification PRO Task Force Report. Value Health. 2009;12(8):1075-1083.

24. Leidy NK. Functional status and the forward progress of merrygo-rounds: toward a coherent analytical framework. Nurs Res. 1994; 43(4):196-202.

25. Leidy NK. Using functional status to assess treatment outcomes. Chest. 1994;106(6):1645-1646.

26. Stull DE, Leidy NK, Jones PW, Stahl E. Measuring functional performance in patients with COPD: a discussion of patient-reported outcome measures. Curr Med Res Opin. 2007;23(11):2655-2665.

27. Leidy NK, Haase JE. Functional performance in people with chronic obstructive pulmonary disease: a qualitative analysis. ANS Adv Nurs Sci. 1996;18(3):77-89.

28. Leidy NK, Haase JE. Functional status from the patient's perspective: the challenge of preserving personal integrity. Res Nurs Health. 1999; 22(1):67-77.

29. Larson JL, Kapella MC, Wirtz S, Covey MK, Berry J. Reliability and validity of the functional performance inventory in patients with moderate to severe chronic obstructive pulmonary disease. J Nurs Meas. 1998;6(1):55-73.

30. Kapella MC, Larson JL, Covey MK, Alex CG. Functional performance in chronic obstructive pulmonary disease declines with time. Med Sci Sports Exerc. 2011;43(2):218-224.

31. Leidy NK, Knebel A. In search of parsimony: reliability and validity of the Functional Performance Inventory-Short Form. Int J Chron Obstruct Pulmon Dis. 2010;5:415-423.

32. Donesky-Cuenco D, Nguyen HQ, Paul S, Carrieri-Kohlman V. Yoga therapy decreases dyspnea-related distress and improves functional performance in people with chronic obstructive pulmonary disease: a pilot study. J Altern Complement Med. 2009;15(3):225-234.

33. Guo AM, Han JN, Leidy NK, Wu ZL, Wang P, Lin YX. Validation of the Chinese version of the Functional Performance Inventory Short Form in patients with chronic obstructive pulmonary disease. J Clin Nurs. 2011;20(11-12):1613-1622. 
34. Wall MP. Predictors of functional performance in community-dwelling people with COPD. J Nurs Scholarsh. 2007;39(3):222-228.

35. Willis G. Cognitive Interviewing. Thousand Oaks, CA: Sage Publications, Inc; 2005.

36. ZuWallack R. How are you doing? What are you doing? Differing perspectives in the assessment of individuals with COPD. COPD. 2007;4(3):293-297.
37. Jehn M, Schmidt-Trucksass A, Meyer A, Schindler C, Tamm M, Stolz D. Association of daily physical activity volume and intensity with COPD severity. Respir Med. 2011;105(12):1846-1852.

38. Rennard S, Decramer M, Calverley PM, et al. Impact of COPD in North America and Europe in 2000: subjects' perspective of Confronting COPD International Survey. Eur Respir J. 2002;20(4): 799-805.

\section{Publish your work in this journal}

The International Journal of COPD is an international, peer-reviewed journal of therapeutics and pharmacology focusing on concise rapid reporting of clinical studies and reviews in COPD. Special focus is given to the pathophysiological processes underlying the disease, intervention programs, patient focused education, and self management protocols.
This journal is indexed on PubMed Central, MedLine and CAS. The manuscript management system is completely online and includes a very quick and fair peer-review system, which is all easy to use. Visit http://www.dovepress.com/testimonials.php to read real quotes from published authors.

Submit your manuscript here: http://www.dovepress.com/international-journal-of-copd-journal 